\title{
Materials for Small Nuclear Reactors and Micro Reactors, Including Space Reactors
}

\author{
SVEN C. VOGEL (1) ${ }^{1}$ MARISA J. MONREAL,${ }^{1}$ \\ and ADITYA P. SHIVPRASAD ${ }^{1}$ \\ 1.-Los Alamos National Laboratory, Los Alamos, NM 87545, USA
}

\begin{abstract}
Small nuclear reactors, including small modular reactors (SMRs) or reactors for space applications, rely on different materials than those typically applied in large-scale nuclear power plants. Examples include molten salts as cooling medium or fuel carrier, metal hydrides as high-temperature moderators, and fuels allowing for higher burnup. All of these also require novel structural materials, for which material interactions have to be understood. Fissionable and fissile materials, such as uranium or plutonium, are rarely considered in materials design other than for nuclear fuels. Similarly, the aspects of radiation damage, occurring during irradiation when a reactor operates, are unique to nuclear materials research. The handling of these materials puts further limitations on the materials science conducted for nuclear materials. All of these issues move research for these materials off the "main stream" of materials science, and cause it to be more easily conducted at national laboratories. However, nuclear reactors offer unique opportunities for carbon-neutral energy generation and have great potential to address if not solve problems arising from global warming. This special topic, sponsored by the TMS Nuclear Materials Committee, focuses on materials research for small nuclear reactors, both experimental and simulation/modeling.
\end{abstract}

The work reported in this special topic covers materials research for various reactor components such as heat pipes and moderators, as well as the nuclear fuel itself. The breadth of material systems discussed include chlorides (solid as well as molten salts), hydrides, carbides, and nitrides, as well as

Sven C. Vogel, Aditya P. Shivprasad and Marisa Monreal are Guest Editors for the Nuclear Materials Committee of TMS and organized the topic Materials for Small Nuclear Reactors and Micro Reactors, including Space Reactors in this issue. high temperature corrosion-resistant metals and alloys. Included papers address phenomena ranging from metal/carbon interactions to hydrogen redistribution in solids to phase diagrams to thermal expansion and other thermophysical properties. Modeling and simulation techniques such as density-functional theory and phase field modeling, and experimental techniques such as neutron diffraction and neutron radiography, are used to advance the state of the art in materials research for nuclear energy generation.

Specifically, Kardoulaki et al. review the interactions of carbon and molybdenum to assess the potential of using molybdenum as a cladding material for heat pipes in a graphite monolith core microreactor design. Trellue et al. report on a comprehensive characterization of yttrium hydride as a solid high temperature moderator material, including the first in situ observation of hydrogen redistribution in response to a temperature gradient by high temperature neutron radiography. Nuclear thermal propulsion for deep space exploration requires research in suitable nuclear fuel systems and Abdul-Jabbar et al. examine the uraniumtitanium-carbon system for this application while Hirschhorn et al. review fuel loss from cermets composed of uranium nitride and a molybdenumtungsten alloy for the same application. Molten salt research is presented by Choi et al., proposing a ranking method for the rate of corrosion of metals in molten fluoride or chloride salts, and Vogel et al. describe the crystal structure evolution of the molten salt reactor fuel system $\mathrm{UCl}_{3}$ from room temperature to melting using high temperature neutron diffraction. Finally, two papers by Parker et al. report on the thorium-uranium-nitrogen system, researched for compact reactors for remote location as well as space applications, and provide neutronic behavior as well as thermophysical properties of the system. 
The breadth of material systems, researched for applications in the main components in novel nuclear reactor concepts and utilizing state of the art simulation and experimental methods, shines a light on current material science problems tackled in this field. The editors hope the readers enjoy these articles and that this special topic issue stimulates further research in this important field of carbon-neutral energy generation.
All titles and authors of the articles are published under the topic "Materials for Small Nuclear Reactors and Micro Reactors, including Space Reactors" in the November 2021 issue (vol. 73, no. 11) of JOM. The articles can be accessed fully via the journal's page at: http://link.springer.com/journal/11837/73/1 1/page/1.

Publisher's Note Springer Nature remains neutral with regard to jurisdictional claims in published maps and institutional affiliations. 